\title{
Response to the Meningococcal Meningitis Epidemic (MME) at Aminu Kano Teaching Hospital, Kano (2008-2009)
}

\author{
Iliyasu G, Lawal H, Habib A G, Hassan-Hanga F, Abubakar I S, Bashir U, Tanko-Yakasai U, Abubakar S, \\ Abba M S, Rano I S, Abdu H, Musa B, Gwarzo G D
}

\begin{abstract}
Departments of Medicine, Paediatrics, Community Medicine, Nursing, Pharmacy and Administration, AKTH, Kano, Nigeria
\end{abstract}

\begin{abstract}
In December 2008 an outbreak of Meningococcal Meningitis swept across sub Saharan Africa with Nigeria, especially its northern states worst affected. The management of Aminu Kano Teaching Hospital constituted an Emergency Preparedness \& Response (EPR) committee. Over the course of 18 weeks from 5 January 2009 to 15 May 2009, AKTH managed 222 cases of suspected meningitis with 14 deaths (case fatality rate [CFR] of $6.3 \%)$. Twenty three per cent (23\%) were microbiologically proven as meningococcal meningitis while $9 \%$ were confirmed to be pneumococcal meningitis. Male to female ratio was 1:1 with most patients (81\%) aged below 14 years. The epidemic peaked in weeks 10 and 13 with 38 admissions in the respective weeks. Meningococcemia with purpura fulminans, post meningitic immune complex cutaneous vasculitis and polyarthritis were observed. Control measures instituted included provision of free ceftriaxone, chemoprophylaxis to contacts, vaccines to staff/families, and creation of dedicated isolation wards. Clinical management guidelines were developed and hospital staffs were also enlightened. Lessons learnt included the difficulty of discriminating between nosocomial transmission and community clusters; relative increase in pneumococcal meningitis during the epidemic; unreliability of penicillin/chloramphenicol; the utility of internet for communication; and the inadequacy of vaccines to meet staff \& public demand.
\end{abstract}

\section{Date Accepted for publication: 18th August 2009 Nig J Med 2009; 428 - 430 \\ Copyright $\odot 2009$ Nigerian Journal of Medicine}

Neisseria meningitidis is a major cause of serious bacterial infection worldwide ${ }^{1 .}$ Meningococcal disease usually presents as meningitis in $80-85 \%$ of cases, whereas in the remaining $15-20 \%$ it presents with pure septicaemia without meningitis. Outbreaks of meningococcal meningitis continue to occur worldwide especially within the 'Meningitis Belt', an area that stretches from Senegal in the west to Ethiopia in the east, ${ }^{2}$ with an estimated total population of 300 million people. Epidemics occur during the dry season ${ }^{3}$ from November to June, perhaps because of diminished nasopharyngeal immunity from repeated upper respiratory tract infections consequent from the dusty winds and cold nights. The transmission of $N$. meningitidis is favoured by overcrowded housing, by large population congregations and displacements. Major epidemics occur in 5-10year cycle, perhaps due to decrease in herd immunity. ${ }^{4}$ Several sero-groups of $\mathrm{N}$. Meningitidis have been identified; however major African epidemics are associated with sero-groups A and $C^{5}$ although sero-group $W 135$ has been associated with outbreaks of considerable size. ${ }^{6}$ The pathogenicity, immunogenicity, and epidemic capabilities differ according to the sero-group. Thus, the identification of the sero-group responsible of a sporadic case is crucial for epidemic containment.

N. meningitidis is transmitted from person to person via droplets of respiratory secretions. The average incubation period is 4 days, ranging between hours to 10 days. The most common symptoms are stiff neck, high fever, photophobia, confusion, headaches and vomiting. Refusal to eat or feed and irritability may occur in children. Even with timely antibiotic therapy, the case fatality rate for meningococcal meningitis is between 5 and $10 \%$, while similar proportions develop transitory allergic complications or long term neurological sequellae, especially deafness. ${ }^{7}$

Nigeria has encountered several outbreaks in the past, with the most devastating being that of 1996 when over 250000 cases and 25000 deaths were registered.

In December 2008 another outbreak started in the sub Saharan Africa with Nigeria and Niger Republics most affected. By the first week of May, 2009, 47,902 cases were recorded with 2,148 deaths from Nigeria alone. 
Estimated $85 \%$ of the Nigerian cases were reported from 16 states of northern Nigeria. Kano state situated between $10^{\circ} 30^{\prime} \mathrm{N}$ and $12^{\circ} 19^{\prime} \mathrm{E}$ and the most populous state in Nigeria, was the worst affected.

The Management of Aminu Kano Teaching Hospital (AKTH), the Regional Teaching Hospital with a catchment population comprising that of Kano and other neighboring states, constituted an Emergency Preparedness and Response (EPR) committee in February 2009. The EPR committee comprised of clinical physicians, pediatricians, community physicians, infectious disease nurses, a microbiologist, pharmacist, statistician and a clerical staff. The 3 main goals of the committee were to contain the epidemic, to ensure cases are effectively managed and to prevent or reduce complications. The committee was able to achieve these goals through sensitization of the hospital community; creation of dedicated isolation units; vaccination of hospital staff/ families and reactive vaccination to the public through collaboration with Kano state Ministry of Health; designing a user friendly case management protocol; provision of free ceftriaxone and chemoprophylaxis to patient's contacts. The Hospital management was fully supportive of the control effort.

During the epidemic which lasted 17 weeks, 222 cases were admitted and managed with 14 deaths (Case Fatality rate [CFR] of $6.3 \%$ ). Fifty one cases $(23 \%)$ were microbiologically proven as meningococcal meningitis while nineteen cases $(9 \%)$ were confirmed to be pneumococcal meningitis. The epidemiological curve (Table II) shows that the epidemic peaked in weeks 10 and 13 with 38 admissions in the respective weeks. The male to female ratio was $1: 1$, and children aged 14 years and below accounted for $81 \%$ of all the cases, (Table III) including two cases just beyond the neonatal period [32 and 40 days, respectively], that presented with widespread purpuric rashes following meningococcemia (Fig.1). All the 222 cases came from the hospital's catchment area suggesting no unusual clustering or spatial pattern (Table IV). Three suspected cases of nosocomial transmissions and 3 clusters were reported during the epidemic, however confirmation was not possible without further molecular studies. Five health care workers were admitted, with no epidemiologic or temporal linkages between them including two doctors, two nurses and one theater attendant. Hospital acquisition could not be excluded.

All the N. meningitidis were sensitive to ceftriaxone while the sensitivity to chloramphenicol and gentamicin was $82 \%$ and $78 \%$ respectively. The S. pneumoniae was $63 \%$ sensitive to both ceftriaxone and chloramphenicol. Neither the N. meningitidis nor the S. pneumoniae were sensitive to penicillin.

Late complications could not be evaluated as follow-up was not always feasible. Though a few cases with neurological complications (seizure, paralysis etc) and immune complex mediated disease were observed, including an adult patient with cutaneous vasculitis and polyarthritis (Fig 2).

\section{Lessons learnt include:}

1) The level of public awareness of infectiousness of meningitis during the epidemics and what to do during the epidemics was very low.

2) Availability of vaccines was limited and could not meet the demand by staff families and neighbours.

3) Relative increase in pneumococcal meningitis during the epidemic

4) Knowledge, attitude and actual implementation of chemoprophylaxis among Health Care Workers [HCWs] were sub-optimal.

5) Unreliability of penicilline/chloramphenicol

6) Difficulty of discriminating between nosocomial transmission and community clusters

7) Utility of internet as a means of communication during such an epidemic

8) Collaborative effort is an effective way of curtailing an epidemic

\section{Recommendations made include:}

1. Active surveillance to monitor communicable disease and early outbreak detection.

2. Establishment of emergency preparation and a response committee to monitor and respond to rising levels of infection.

3. Public education about early symptoms/dispelling misconception regarding transmission should be improved by the hospital as a community service.

4. An intercom may be provided outside isolation wards so that relations can visit and speak with patients in isolation there by risk of hospital transmission

5. Policy guidelines regarding the quantities and type of vaccine- preparation that would ensure timely protection should be advocated for.

6. Quick and early mass vaccination is to be encouraged. 
7. The facility should work with national institutions to procure some stock of quadrivalent vaccines in case of non-A/non-C MME in the future.

8. Simplified treatment protocols due to potential shortages, logistic difficulties, and high incidence.

9. Timely supply of essential and appropriate drugs preferably free or at subsidized prices.

10. Practitioners should be enlightened to provide optimal (meningitic) doses during epidemics

11. Adequately equipping the laboratory to precisely identify the organism including the serotype

12. Follow-up of patients after discharge through effective collaboration between community medicine, community nursing and social welfare departments will help in alleviating and managing complications.

13. Requirements per patient (drugs, vaccines, consumables) derived from this MME can be used to forecast in the future.

14. EPR activities may be broadened to include emergency and natural disaster management and response.

15. Communication with state $\mathrm{MOH}$ was good and it should be sustained and improved

\section{Fig.1: Purpura Fulminans in a Neonate}

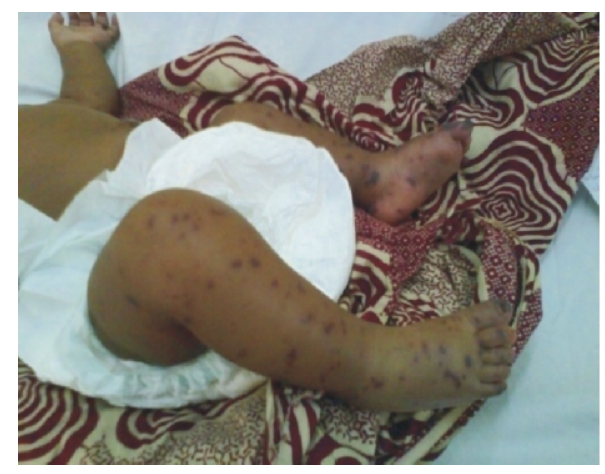

Fig. 2 : Post Meningococcal Meningitis Immune Complex Mediated Polyarthritis And Vasculitis In An Adult
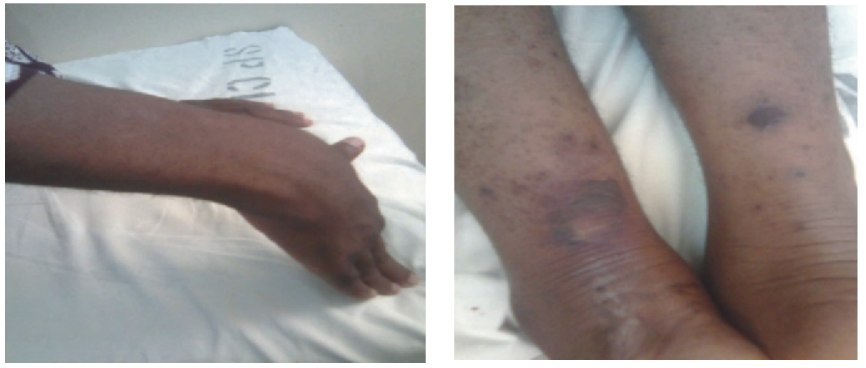

\section{Fig 3 The Epidemiology Curve}

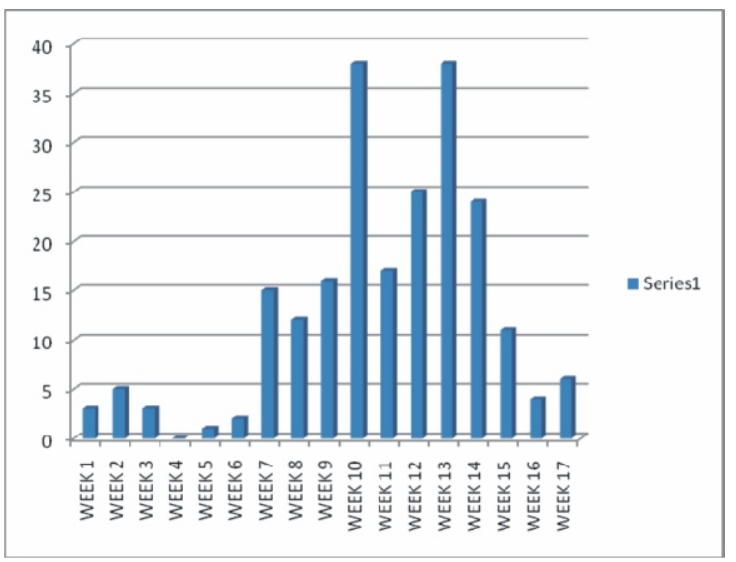

Table III: Age/Sex Distribution of Patients Admitted into Various Wards of the Hospital

\begin{tabular}{|l|l|l|l|}
\hline Age(Years) & Male & Female & Total $(\%)$ \\
\hline $0-9$ & 76 & 80 & $156(70.0 \%)$ \\
\hline $10-19$ & 12 & 14 & $26(11.8 \%)$ \\
\hline $20-29$ & 9 & 7 & $16(7.3 \%)$ \\
\hline $30-39$ & 11 & 6 & $17(7.7 \%)$ \\
\hline $40-49$ & 2 & 3 & $5(2.2 \%)$ \\
\hline $50-59$ & - & - & - \\
\hline $60-69$ & 1 & - & $1(0.5 \%)$ \\
\hline $70-79$ & 0 & 1 & $1(0.5 \%)$ \\
\hline TOTAL & 106 & 108 & $220(100.0 \%)$ \\
\hline
\end{tabular}

\section{Acknowledgement}

We would like to thank the Chief Medical Director Dr Abdulhamid I Dutse, the Chairman Medical Advisory Committee Dr Aminu Zakari, Kano State Ministry of Health and all the managing physicians and medical records staff for help and support in carrying out this task.

\section{References}

1. Rosenstein NE, Perkins BA, Stephens DS et al. Meningocococcal disease. N Engl J Med 2001; 344:13781388

2. Lapeyssonie L. La m'eningite ce're'brospinale en Afrique. Bull WId HIth Org 1963;28:3-114

3. Greemwood BM, Bradley AK, Cleland PG et al. An epidemic of meningococcal infection at Zaria, Northern Nigeria. General epidemiological features. Trans Roy Soc Trop Med Hyg 1979; 73:557-562

4. Greenwood BM. Meningococcal meningitis in Africa. Trans Roy Soc Trop Med Hyg 1999; 93:341-353

5. Principles of Medicine in Africa. Edited by Eldryd Parry et al Third edition. Cambridge University Press 2004

6. Decosas J, Koama J-BT. Chronicle of an outbreak foretold; Meningococcal meningitis W135 in Burkina Faso. Lancet Infect Dis 2002;ii:763-765

7. Smith AW, Bradley AK, Wall RA et al. Sequelae of epidemic meningococcal meningitis in Africa. Trans Roy Soc Trop Med Hyg 1988; 312-3 